\title{
Cross-Correlation of the Extragalactic Gamma-ray Background with Luminous Red Galaxies
}

\author{
Masato Shirasaki \\ National Astronomical Observatory of Japan, Mitaka, Tokyo 181-8588, Japan* \\ Shunsaku Horiuchi \\ Center for Neutrino Physics, Department of Physics, \\ Virginia Tech, Blacksburg, Virginia 24061, USA Đ \\ Naoki Yoshida \\ Department of Physics, University of Tokyo, Tokyo 113-0033, Japan \\ Kavli Institute for the Physics and Mathematics of the Universe (WPI), \\ University of Tokyo, Kashiwa, Chiba 277-8583, Japan and \\ CREST, Japan Science and Technology Agency, 4-1-8 Honcho, Kawaguchi, Saitama, 332-0012, Japan 团
}

\begin{abstract}
Measurements of the cross-correlation between the extragalactic gamma-ray background (EGB) and large-scale structure provide a novel probe of dark matter on extragalactic scales. We focus on luminous red galaxies (LRGs) as optimal targets to search for the signal of dark matter annihilation. We measure the cross-correlation function of the EGB taken from the Fermi Large Area Telescope with the LRGs from the Sloan Digital Sky Survey. Statistical errors are calculated using a large set of realistic mock LRG catalogs. The amplitude of the measured cross-correlation is consistent with null detection. Based on an accurate theoretical model of the distribution of dark matter associated with LRGs, we exclude dark matter annihilation cross-sections over $\langle\sigma v\rangle=3 \times 10^{-25}-10^{-26} \mathrm{~cm}^{3} \mathrm{~s}^{-1}$ for a $10 \mathrm{GeV}$ dark matter. We further investigate systematic effects due to uncertainties in the Galactic gamma-ray foreground emission, which we find to be an order of magnitude smaller than the current statistical uncertainty. We also estimate the contamination from astrophysical sources in the LRGs by using known scaling relations between gamma-ray luminosity and star-formation rate, finding them to be negligibly small. Based on these results, we suggest that LRGs remain ideal targets for probing dark matter annihilation with future EGB measurement and galaxy surveys. Increasing the number of LRGs in upcoming galaxy surveys such as LSST would lead to big improvements of factors of several in sensitivity.
\end{abstract}

\section{INTRODUCTION}

Dark matter $(\mathrm{DM})$ is invoked to explain a broad range of astronomical and cosmological observations. DM constitutes $\sim 85 \%$ of the matter content of the Universe, and thus plays an essential role in the development of the rich structure of the Universe, such as galaxies and clusters of galaxies. Although the nature of DM still remains unknown, weakly-interacting massive particles (WIMPs) are among the most attractive particle physics candidates. In the WIMP hypothesis, some unknown weakly-interacting particle (DM) is initially in thermal equilibrium in the early Universe. The time evolution of the mean number density of the particles is determined by the DM annihilation rate and the expansion rate of the Universe. Interestingly, the observed abundance of DM can be naturally explained by WIMPs with mass in the range of $10 \mathrm{GeV}-10 \mathrm{TeV}$ if their annihilation cross-section is of the order the cross-section for weak interactions [1].

Several "local" gamma-ray measurements have already

\footnotetext{
* masato.shirasaki@nao.ac.jp

$\dagger$ horiuchi@vt.edu

$\ddagger$ naoki.yoshida@ipmu.jp
}

probed interesting parameter ranges of annihilating DM. Such probes rely on searching for gamma rays from nearby regions where the DM density is expected to be large, since the production rate of the gamma rays is proportional to the DM density squared. Thus, Milky Way satellite galaxies [2 [4] or the Galactic center [5, 6] are considered to be the most promising targets for searching for the signal of DM annihilation.

Recently, the cross-correlation of the extragalactic gamma-ray background (EGB) with large-scale structure (LSS) has been proposed as a complementary probe 7 11]. In addition to known gamma-ray sources such as blazers, misaligned AGNs, and star-forming galaxies, the DM distributed on extragalactic scales should contribute to the observed EGB. Although the mean EGB intensity can be explained by unresolved astrophysical sources [12], the anisotropy in the diffuse gamma-ray sky contains, in principle, rich information about any unresolved DM source contributions (e.g., see Ref [13] for review). This is because the DM density distribution in the Universe is expected to be highly inhomogeneous due to the nonlinear gravitational growth of structure. Therefore, any observational tracers of the DM distribution should correlate with the extragalactic gamma-ray map. In fact, the latest gamma-ray data taken from the Fermi Large Area Telescope (LAT) have already shown a positive cor- 
relation with several known tracers of DM distribution such as nearby galaxies, radio galaxies, and quasars 14], as well as the reconstructed mass distribution from weak lensing effect on the cosmic microwave background 15].

Given the successful detections of the cross-correlation between the EGB and LSS [14, 15], it is important and timely to identify optimal targets to possibly detect the signal of DM annihilation on a statistical basis. Lowredshift galaxies are among the most promising targets for cross-correlation, but it is thought to be difficult to disentangle the contributions from astrophysical sources and DM annihilation [16]. In order to break the degeneracy, Refs 10, 11] proposed a tomographic approach, i.e., measuring the cross-correlation in separate bins in redshift and/or energy.

In the present paper, we propose an alternative optimal target to search for DM annihilation through crosscorrelation studies. We consider the following three conditions: (i) the targets must have accurate redshift information so that a volume limited sample can be generated, (ii) any intrinsic gamma-ray luminosity from astrophysical sources should be small, and (iii) the statistical properties of the host DM can be determined observationally. Among the possible candidates, we focus on the luminous red galaxies (LRGs) selected from the Sloan Digital Sky Survey (SDSS). LRGs have the advantages of having accurate spectroscopic redshifts, low star-formation rates, and a well-constrained relation of their underlying DM distribution through clustering measurements and weak lensing analyses. We thus measure the cross-correlation of the EGB with the LRGs to search for DM annihilation signatures. We calculate statistical errors using realistic mock LRG catalogs, and study in detail possible systematic effects on the cross-correlation measurement. In particular, we estimate the systematic error due to the uncertainty in the Galactic gamma-ray foreground modeling and star-forming activity in the LRGs.

The paper is organized as follows. In Section II] we describe the contribution to the EGB from DM annihilation. We also present a theoretical model of crosscorrelation of the EGB with LRGs. In Section III, we describe the target galaxies and the gamma-ray data. The details of the cross-correlation analysis are provided in Section[IV In Section V], we show the result of our crosscorrelation analysis, and derive constraints on the DM annihilation cross-section. Finally, we investigate systematic uncertainties of our measurement in Section VI. Concluding remarks and discussions are given in Section VII. Throughout the paper, we use the standard cosmological parameters $H_{0}=100 h \mathrm{~km} \mathrm{~s}^{-1}$ with $h=0.7$, $\Omega_{\mathrm{m} 0}=0.279$, and $\Omega_{\Lambda}=0.721$.

\section{DARK MATTER ANNIHILATION}

The contribution of DM annihilation to the EGB intensity $I_{\gamma}$ (the number of photons per unit energy, area, time, and solid angle) is given by,

$$
\begin{aligned}
E_{\gamma} I_{\gamma}=\frac{c}{4 \pi} \int \frac{\mathrm{d} z}{H(z)(1+z)^{4}} E_{\gamma}^{\prime} \frac{\mathrm{d} N_{\gamma}}{\mathrm{d} E_{\gamma}^{\prime}} \frac{\langle\sigma v\rangle}{2} \\
\times\left[\frac{\rho_{\mathrm{dm}}(\boldsymbol{x} \mid z)}{m_{\mathrm{dm}}}\right]^{2} e^{-\tau\left(E_{\gamma}^{\prime}, z\right),}
\end{aligned}
$$

where $E_{\gamma}$ is the observed gamma-ray energy, $E_{\gamma}^{\prime}=$ $(1+z) E_{\gamma}$ is the energy of the gamma ray at redshift $z, H(z)=H_{0}\left[\Omega_{\mathrm{m} 0}(1+z)^{3}+\Omega_{\Lambda}\right]^{1 / 2}$ is the Hubble parameter in a flat Universe, and the exponential factor takes into account the effect of gamma-ray attenuation during propagation owing to pair creation on diffuse extragalactic photons. We adopt the gamma-ray optical depth $\tau\left(E_{\gamma}^{\prime}, z\right)$ from Ref. [17]. The physics of DM is contained in $\mathrm{d} N_{\gamma} / \mathrm{d} E_{\gamma}$, the gamma-ray spectrum per annihilation; $\langle\sigma v\rangle$, the annihilation cross-section times the relative velocity averaged with the velocity distribution function; $\rho_{\mathrm{dm}}(\boldsymbol{x} \mid z)$, the DM mass density distribution at redshift $z$ as a function of spatial coordinate $\boldsymbol{x}$; and $m_{\mathrm{dm}}$, the DM particle mass.

For the gamma-ray spectrum per annihilation $\mathrm{d} N_{\gamma} / \mathrm{d} E_{\gamma}$, we adopt two characteristic spectra corresponding to annihilation with $100 \%$ branching ratios to $b \bar{b}$ and $\tau^{+} \tau^{-}$final states. We use the PPPC4DMID package [18] that is based on PYTHIA (v8.135) and HERWIG (v6.510) event generators. The spectra are dominated by emission from the decay of neutral pions. These are primary gamma-ray emissions, and should be distinguished from secondary emission that results from interactions of the annihilation products with the environment. Throughout this paper, we do not include secondary emissions. Secondary emissions are only important for annihilation products propagating in regions of high baryon density, e.g., in and around galactic disks. This makes them interesting from the perspective of depending strongly on the astrophysical environment, but they are a minor contribution to the total gamma-ray yield from halos.

\section{A. Cross-correlation with galaxy distribution}

Since the contribution from DM annihilation scales with the DM density squared, any tracer of the DM density distribution should correlate with the gamma-ray intensity distribution. The galaxy distribution over the sky is among the most promising tracers of the DM density distribution in the Universe. While galaxies themselves are biased tracers of DM, the statistical properties of galaxy clustering are well understood and thus can be incorporated theoretically in the so-called halo model approach, where one assumes that all the matter are contained in spherical DM halos. Within the halo model approach, one can relate the galaxy distribution to the DM density distribution by introducing a halo occupation distribution (HOD). The HOD $\left\langle N_{\text {gal }} \mid M\right\rangle$ describes the mean number of galaxies in a host halo of mass of 
$M$. For a given $\left\langle N_{\text {gal }} \mid M\right\rangle$ at redshift $z$, the cross-power spectrum of galaxy overdensity $\delta_{\text {gal }}$ and DM overdensity squared $\delta^{2}$ can be expressed as [10],

$$
\begin{aligned}
P_{\mathrm{gal}, \delta^{2}}(k, z) & =P_{\mathrm{gal}, \delta^{2}}^{1 h}(k, z)+P_{\mathrm{gal}, \delta^{2}}^{2 h}(k, z), \\
P_{\mathrm{gal}, \delta^{2}}^{1 h}(k, z)= & \int \mathrm{d} M \frac{\mathrm{d} n}{\mathrm{~d} M} \frac{\mathcal{J}(z, M)}{\bar{\rho}_{\mathrm{dm}}^{2}(z)} \frac{\left\langle N_{\mathrm{gal}} \mid M\right\rangle}{\bar{n}_{\mathrm{gal}}(z)} \\
\times & \tilde{u}_{\mathrm{gal}}(k \mid z, M) \tilde{u}_{\delta^{2}}(k \mid z, M), \\
P_{\mathrm{gal}, \delta^{2}}^{2 h}(k, z)= & {\left[\int \mathrm{d} M \frac{\mathrm{d} n}{\mathrm{~d} M} b_{h}(z, M) \frac{\left\langle N_{\mathrm{gal}} \mid M\right\rangle}{\bar{n}_{\text {gal }}(z)} \tilde{u}_{\text {gal }}(k \mid z, M)\right] } \\
\times & {\left[\int \mathrm{d} M \frac{\mathrm{d} n}{\mathrm{~d} M} b_{h}(z, M) \frac{\mathcal{J}(z, M)}{\bar{\rho}_{\mathrm{dm}}^{2}(z)} \tilde{u}_{\delta^{2}}(k \mid z, M)\right] } \\
& \times P_{\text {lin }}(k, z),
\end{aligned}
$$

where $P_{\text {lin }}(k, z)$ is the linear matter power spectrum at $z, \mathrm{~d} n / \mathrm{d} M$ is the halo mass function, $b_{h}(z, M)$ represents the linear halo bias, and $\mathcal{J}(z, M)$ is the volume integral of DM density squared in the spherical halo. We set the minimum halo mass to be $10^{-6} h^{-1} M_{\odot}$ in the integral in Eqs. (3) and (4). Note that the integral is insensitive to the minimum halo mass as long as it is set to be $\lesssim 10^{10} h^{-1} M_{\odot}$ due to the functional form of the HOD for the LRGs. In this paper, we define the term $\mathcal{J} \tilde{u}_{\delta^{2}}$ as the fourier transform of $\rho_{\mathrm{dm}}^{2}(\boldsymbol{x})$ inside a halo, while $\left\langle N_{\text {gal }} \mid M\right\rangle \tilde{u}_{\text {gal }}$ corresponds to the Fourier transform of galaxy distribution in a host halo. The mean number density of galaxies $\bar{n}_{\text {gal }}(z)$ is then given by,

$$
\bar{n}_{\text {gal }}(z)=\int \mathrm{d} M \frac{\mathrm{d} n}{\mathrm{~d} M}\left\langle N_{\text {gal }} \mid M\right\rangle .
$$

To calculate $P_{\text {gal }, \delta^{2}}$, we adopt the model of halo mass function and linear halo bias in Refs. 19, 20]. We calculate $\tilde{u}_{\delta^{2}}$ following Ref. [21]. Apart from the HOD, the largest uncertainty in our halo model calculation is in the factor $\mathcal{J}$. In particular, the amplitude of $\mathcal{J}$ is sensitive to the amount of substructures in a halo and thus is still poorly known even for massive galaxies or cluster-size halos. In practice, the $\mathcal{J}$ factor can be expressed as,

$$
\mathcal{J}(z, M)=\left(1+b_{s h}(z, M)\right) \int \mathrm{d} V \rho_{h}^{2}(r \mid z, M),
$$

where $\rho_{h}$ represents the density profile of a spherical halo and $b_{s h}$ is the boost factor, which describes the effective "boost" to the amplitude of the DM density squared owing to subhalos. For the smooth component of the density profile, we adopt the NFW profile [22] with concentrations as given in Ref [23]. For the boost factor $b_{s h}$, we consider two extreme phenomenological models by Refs. 24, 25]. In the first, subhalo properties are scaled as power-laws and extrapolated many orders of magnitudes to the smallest subhalos $\left(10^{-6} M_{\odot}\right)$ [24]. This procedure yields large boost factors, but it is rather uncertain whether such extrapolations are valid to such small halos. We thus adopt the model as an optimistic scenario. In the second, the halo concentration is extrapolated by a relation that flattens at small halo masses, yielding smaller boosts [25]. We treat this as a conservative scenario, since the concentration-mass relation derives from field halos rather than subhalos; for a given mass, subhalos are expected to be more concentrated than field halos, hence the boost factor should be higher. Ref. [26] estimates the effect is a factor of $2-5$ increase in the boost factors.

Observations of a large number of galaxies enable us to constrain the HOD fairly precisely. The HOD of luminous red galaxies has already been studied with number counts [27], spatial clustering [28], and galaxy-galaxy lensing analysis [29]. A popular HOD model is given by,

$$
\begin{aligned}
\left\langle N_{\text {gal }} \mid M\right\rangle & =\left\langle N_{\text {cen }} \mid M\right\rangle\left(1+\left\langle N_{\text {sat }} \mid M\right\rangle\right), \\
\left\langle N_{\text {cen }} \mid M\right\rangle & =\frac{1}{2}\left[1+\operatorname{erf}\left(\frac{\log M-\log M_{\text {min }}}{\sigma_{\log M}}\right)\right], \\
\left\langle N_{\text {sat }} \mid M\right\rangle & =\left(\frac{M-M_{\text {cut }}}{M_{1}}\right)^{\alpha},
\end{aligned}
$$

where $\left\langle N_{\text {cen }} \mid M\right\rangle$ represents the HOD of central galaxies which resides at the halo center and $\left\langle N_{\text {sat }} \mid M\right\rangle$ represents the contribution from satellite galaxies. In this paper, we adopt the values of five parameters $\left(M_{\min }, \sigma_{\log M}, M_{\text {cut }}\right.$, $\left.M_{1}, \alpha\right)$ in Ref. [27]. We also assume that the satellite galaxy distribution follows the DM distribution within a halo. Thus, $\left\langle N_{\text {gal }} \mid M\right\rangle \tilde{u}_{\text {gal }}$ is set to be,

$$
\begin{aligned}
\left\langle N_{\text {gal }} \mid M\right\rangle \tilde{u}_{\text {gal }}=\langle & N_{\text {cen }}|M\rangle \\
& +\left\langle N_{\text {cen }} \mid M\right\rangle\left\langle N_{\text {sat }} \mid M\right\rangle \tilde{u}_{\text {dm }},
\end{aligned}
$$

where $\tilde{u}_{\mathrm{dm}}$ is the Fourier transform of $\rho_{h} / M$.

Using the Limber approximation [30] and Eq. (1), we can calculate the angular cross-power spectrum of the LRG surface density and extragalactic gamma-rays emitted through DM annihilation as,

$$
\begin{aligned}
C_{\mathrm{gal}, \mathrm{dm}}(\ell)=\int \frac{\mathrm{d} \chi}{\chi^{2}} & W_{\mathrm{gal}}(\chi) W_{\mathrm{dm}}(\chi) \\
& \times P_{\text {gal }, \delta^{2}}\left(k=\frac{\ell+1 / 2}{\chi}, z(\chi)\right)
\end{aligned}
$$

where $P_{\text {gal }, \delta^{2}}$ is given by Eq. (2) and $W_{\text {gal }}$ is defined as the normalized redshift distribution of galaxies [10]. Here, we define $W_{\mathrm{dm}}$ as,

$$
\begin{aligned}
I_{\gamma}(\hat{\boldsymbol{n}})= & \int \mathrm{d} \chi W_{\mathrm{dm}}(\chi)\left(\frac{\rho_{\mathrm{dm}}(\chi \hat{\boldsymbol{n}}, \chi)}{\bar{\rho}_{\mathrm{dm}}}\right)^{2} \\
W_{\mathrm{dm}}(\chi)=\frac{\langle\sigma v\rangle}{8 \pi} & \left(\frac{\bar{\rho}_{\mathrm{dm}}}{m_{\mathrm{dm}}}\right)^{2}(1+z)^{3} \\
& \times \int_{E_{\gamma, \min }}^{E_{\gamma, \max }} \mathrm{d} E_{\gamma} \frac{\mathrm{d} N_{\gamma}}{\mathrm{d} E_{\gamma}^{\prime}} e^{-\tau\left(E_{\gamma}^{\prime}, z\right)}
\end{aligned}
$$

where $E_{\gamma}^{\prime}=(1+z) E_{\gamma}$ [see Eq. (1)] and $I_{\gamma}(\hat{\boldsymbol{n}})$ is the gamma-ray intensity integrated over a given energy range of $E_{\gamma, \min }$ to $E_{\gamma, \max }$ along a direction $\hat{\boldsymbol{n}}$. In practice, a more direct observable is the two-point crosscorrelation function of the surface galaxy density $\Sigma_{\text {gal }}(\hat{\boldsymbol{n}})$ 


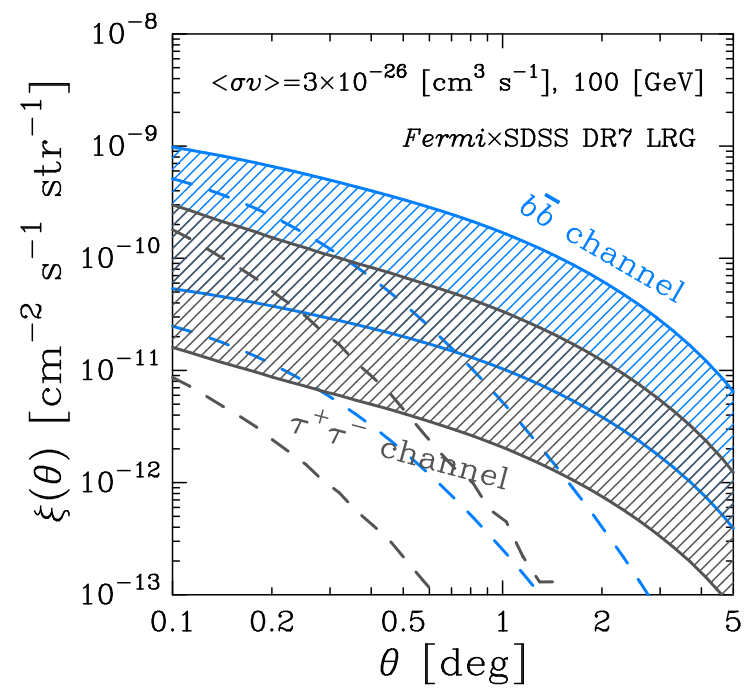

FIG. 1. The cross-correlation function of the EGB with LRGs. We plot the correlation expected from DM annihilation. We assume a DM particle mass of $100 \mathrm{GeV}$ and canonical cross-section of $\langle\sigma v\rangle=3 \times 10^{-26} \mathrm{~cm}^{3} / \mathrm{s}$. The different colors represent different annihilation channels: $b \bar{b}$ (cyan) and $\tau^{+} \tau^{-}$channel (gray). For each colored line, the shaded band indicates our conservative estimate of the theoretical uncertainty caused by the subhalo boost factor model. The solid line shows the total correlation function while the dashed line represents the so-called one-halo term (see text).

and gamma-ray intensity $I_{\gamma}(\hat{\boldsymbol{n}})$. The two-point crosscorrelation function $\xi(\theta)$ can be calculated from the angular power spectrum by the following equations:

$$
\begin{aligned}
\xi(\theta) & =\left\langle I_{\gamma}(\hat{\boldsymbol{n}}) \Sigma_{\text {gal }}(\hat{\boldsymbol{n}}+\boldsymbol{\theta})\right\rangle-\left\langle I_{\gamma}\right\rangle\left\langle\Sigma_{\text {gal }}\right\rangle \\
& =\sum_{\ell} \frac{2 \ell+1}{4 \pi} C_{\text {gal }, \mathrm{dm}}(\ell) P_{\ell}(\cos \theta),
\end{aligned}
$$

where $P_{\ell}(\cos \theta)$ is the Legendre polynomial.

Figure 1 shows our benchmark model of $\xi(\theta)$. We consider two representative annihilation channels $(b \bar{b}$ and $\tau^{+} \tau^{-}$) for a $100 \mathrm{GeV} \mathrm{DM}$ with a thermal cross-section $\langle\sigma v\rangle=3 \times 10^{-26} \mathrm{~cm}^{3} / \mathrm{s}$. We also show our conservative treatment of the theoretical uncertainty originating from the models of boost factor $b_{s h}$. Hence, the expected correlation for each channel lies in the shaded region in Fig. 1. The solid line shows the sum of the one-halo term and the two-halo term, whereas the dashed line represents the contribution of the one-halo term alone. In our theoretical model, the uncertainty of the boost factor causes an uncertainty in $\xi(\theta)$ of a factor of $\sim 10$. Also, the one-halo term dominates on scale of $\lesssim 0.2 \mathrm{deg}$, while the two-halo term induces significant correlations at $\gtrsim 1$ deg.

\section{DATA}

\section{A. SDSS DR7 LRG}

The Slone Digital Sky Survey (SDSS) provides the largest sample of LRGs to date. The SDSS has imaged the sky at high Galactic latitude in five passbands $u, g, r, i$, and $z$ [31, 32], taken by the $2.5 \mathrm{~m}$ APO telescope [33]. The process [34 37] and calibration 38 40. of observed images enable to select galaxies [41, 42] and quasars [43] spectroscopically with twin fiber-fed double spectrographs. Targets are assigned to plug plates according to the tiling algorithm in Ref 44]. The SDSS I/II imaging surveys were completed with a seventh data release (DR7) 45].

In Ref [41], the authors developed an algorithm for selecting LRGs that we use in the present paper as tracers of the underlying matter in and around massive DM halos. We use the publicly available "Bright" LRG catalog made by Ref [46]. The sample consists of 30,272 LRGs with absolute magnitude $-23.2<M_{g}<-21.2$ in the redshift range of $0.16<z<0.44$. They are populated over about 7,200 square degrees in the Northern Galactic Cap with nearly constant comoving number density. The two-point correlation function of the LRG sample has been studied in Ref [46]. We note that possible systematic effects on the correlation analysis is expected to be smaller than the sample variance estimated from mock LRG catalogues (see the appendix in Ref [46] for details).

Note that Ref 14] also considered the cross correlation of the EGB with LRGs. The LRGs used in this paper are the spectroscopic LRG sample, while Ref [14] considered the photometric LRGs from SDSS data release 8. We refer the former as spec-LRGs, while the latter is denoted as photo-LRGs. The advantages to use spec-LRGs in our paper are as follows: (i) the accurate redshift distribution by spectroscopic redshift, (ii) the observationally constrained HOD, and (iii) a large number of mock catalogs. According to the accurate redshift distribution and the well-constrained HOD, the spec-LRGs are known for having relatively lower redshift than photo-LRGs and the typical halo mass of $\sim 10^{14} h^{-1} M_{\odot}$. The redshift range of the photo-LRGs in Ref [14] is $0.45<z<0.65$ with a mean redshift of 0.5 , while the spec-LRGs locate at $0.16<z<0.44$. Since more massive DM halos at lower redshift would have larger contribution to the EGB through DM annihilation, we decide to use the spec-LRGs for cross-correlation analysis with EGB. Furthermore, compared to Ref [14], we improve the crosscorrelation analysis by using accurate covariances measured directly from a large set of mock catalogues of the spec-LRGs.

\section{B. Extragalactic gamma-ray background}

The Fermi satellite provides a full-sky coverage of the GeV gamma-ray sky. We utilize publicly available Fermi- 
LAT Pass 7 Reprocessed photon data taken from August 2008 to December 2014. We divide the SDSS LRGs survey patch into two square ROIs, left and right, each covering $80^{\circ}$ on a side. Using the Fermi Tools version v10r0p5, we use the gtmktime tool to reduce the photon data by removing data taken during nonsurvey modes and removing times when the satellite rocking angle exceeds $52^{\circ}$ with respect to the zenith. These produce a photon sample suitable for analyses. We work with ULTRACLEAN-class photons, which are events that pass the most stringent quality cuts, and we use photons between 1 and $500 \mathrm{GeV}$ in energy. As described below, both choices help adopt a small point source mask. With the gtbin tool, we bin photons into $0.2^{\circ} \times 0.2^{\circ}$ pixels and 30 energy bins equally spaced logarithmically. These binnings are the recommended values by the Fermi collaboration to ensure reasonable analysis outcomes. We generate exposure maps using the standard gtltcube and gtexpcube2 tools, using the P7REP_ULTRACLEAN_V15 instrument response function.

We obtain the extragalactic diffuse photons separately for each ROI. First, we perform a likelihood fit of the reduced photon counts cube using the gtlike tool and find the best fitting Galactic diffuse emission model. We include the following templates in the fits: all the known point and diffuse sources in the 3FGL catalog, a template for the Galactic diffuse foreground emission, and a template for the isotropic emission. As described in Section VIA, we consider multiple templates for the Galactic diffuse emission model, whose uncertainties can dominate analyses of residuals. Only the normalizations of the Galactic diffuse and isotropic emission templates are varied in the fits. We have confirmed that the best-fit Galactic diffuse maps between two ROIs change by $1 \%$ or less and the difference is unimportant for our analysis. Next, we create a model counts cube for the Galactic emission model, as well as any known diffuse sources in the 3FGL catalog. These model counts cubes are subtracted from the raw photon counts cube. Finally, we mask all known point sources within the ROI. Our default mask size is $1^{\circ}$ around each point source, and is motivated by the behavior of the point spread function (PSF): the $68 \%$ containment angle is $\sim 0.9^{\circ}$ at $1 \mathrm{GeV}$ and $\sim 0.26^{\circ}$ at $10 \mathrm{GeV}$ when both front and back conversion tracks are included. Since most point sources have steeply falling spectra, their emissions are dominated by lower energy photons, and we conservatively chose a $1^{\circ}$ mask. We also test that our results are unaffected by changing the mask size to $2^{\circ}$.

For our cross-correlation analysis, we use both front and back conversion tracks. To reduce the impact of the Galactic emission on our analysis, we apply a Galactic latitude cut $|b|>20^{\circ}$ [47]. Moreover, we also exclude the region associated to the Fermi Bubbles and the Loop I structure by applying a Galactic longitude cut $50^{\circ}<\ell<$ $280^{\circ}$ [48].

\section{CROSS-CORRELATION OF EGB WITH LRG}

In order to calculate the cross-correlation of the EGB with the LRGs, we use the following estimator:

$$
\begin{aligned}
\xi(\theta)= & {\left[\sum_{i}^{N_{\text {pix }}} \sum_{j}^{N_{\text {pix }}}\left(I_{\gamma}^{\text {obs }}\left(\phi_{i}\right)-I_{\gamma}^{\text {gm }}\left(\phi_{i}\right)\right) \Sigma_{\text {gal }}\left(\phi_{j}\right) \Delta_{\theta}\left(\phi_{i}-\phi_{j}\right)\right] } \\
& \times\left[\sum_{i}^{N_{\text {pixel }}} \sum_{j}^{N_{\text {pixel }}} \Delta_{\theta}\left(\phi_{i}-\phi_{j}\right)\right]^{-1}
\end{aligned}
$$

where $N_{\text {pix }}$ is the number of pixels in the gamma-ray intensity map, $\Sigma_{\text {gal }}\left(\phi_{i}\right)$ is the overdensity of galaxies in pixel $i, I_{\gamma}^{\text {obs }}\left(\phi_{i}\right)$ is the observed gamma-ray intensity in pixel $i$, and $I_{\gamma}^{\mathrm{gm}}\left(\phi_{i}\right)$ is the contribution from the Galactic emission model estimated using the Fermi-LAT diffuse template and detector modeling. In Eq. (15), we define the function $\Delta_{\theta}(\phi)=1$ for $\theta-\Delta \theta / 2 \leq \phi \leq \theta+\Delta \theta / 2$ and zero otherwise. We have checked that our estimator is consistent with a zero signal when applied to mock LRG catalogues and the observed gamma-ray intensity map.

For binning in angular separation $\theta$, we use 40 bins linearly spaced in $\Delta \theta=0.5 \mathrm{deg}$. In calculating Eq. (15), we do not perform any corrections to the effect of PSF of the Fermi-LAT detector. In the present paper, we take the PSF smearing into account in theoretical models.

We then estimate the covariance matrix $C_{i j}$ of the estimator Eq. (15) by,

$$
C_{i j}=\frac{1}{N_{\mathrm{re}}-1} \sum_{r}\left(\xi^{r}\left(\theta_{i}\right)-\bar{\xi}\left(\theta_{i}\right)\right)\left(\xi^{r}\left(\theta_{j}\right)-\bar{\xi}\left(\theta_{j}\right)\right),
$$

where $\xi^{r}\left(\theta_{i}\right)$ is the estimator for the $i$-th angular bin obtained from the $r$-th realization, and $N_{\text {re }}=160$ is the number of randomized catalogues. The ensemble average of the $i$-th angular bin over 160 realizations, $\bar{\xi}\left(\theta_{i}\right)$, is simply given by,

$$
\bar{\xi}\left(\theta_{i}\right)=\frac{1}{N_{\mathrm{re}}} \sum_{r} \xi^{r}\left(\theta_{i}\right) .
$$

In this paper, we consider two sets of random catalogues: mock LRG catalogues created from "Las Damas" Nbody simulation 11 (McBride et al, in prep) and randomized gamma-ray count maps generated by the real data through Poisson processes. The former provides random realizations of spatial clusterings of LRGs, while the latter can be used to simulate the photon count noise. To simulate the photon count noise, we generate 160 randomized count maps in the same way as shown in Ref. [49]. According to the estimated exposure over the SDSS region, we derive the gamma-ray intensity for

1 http://lss.phy.vanderbilt.edu/lasdamas/ 

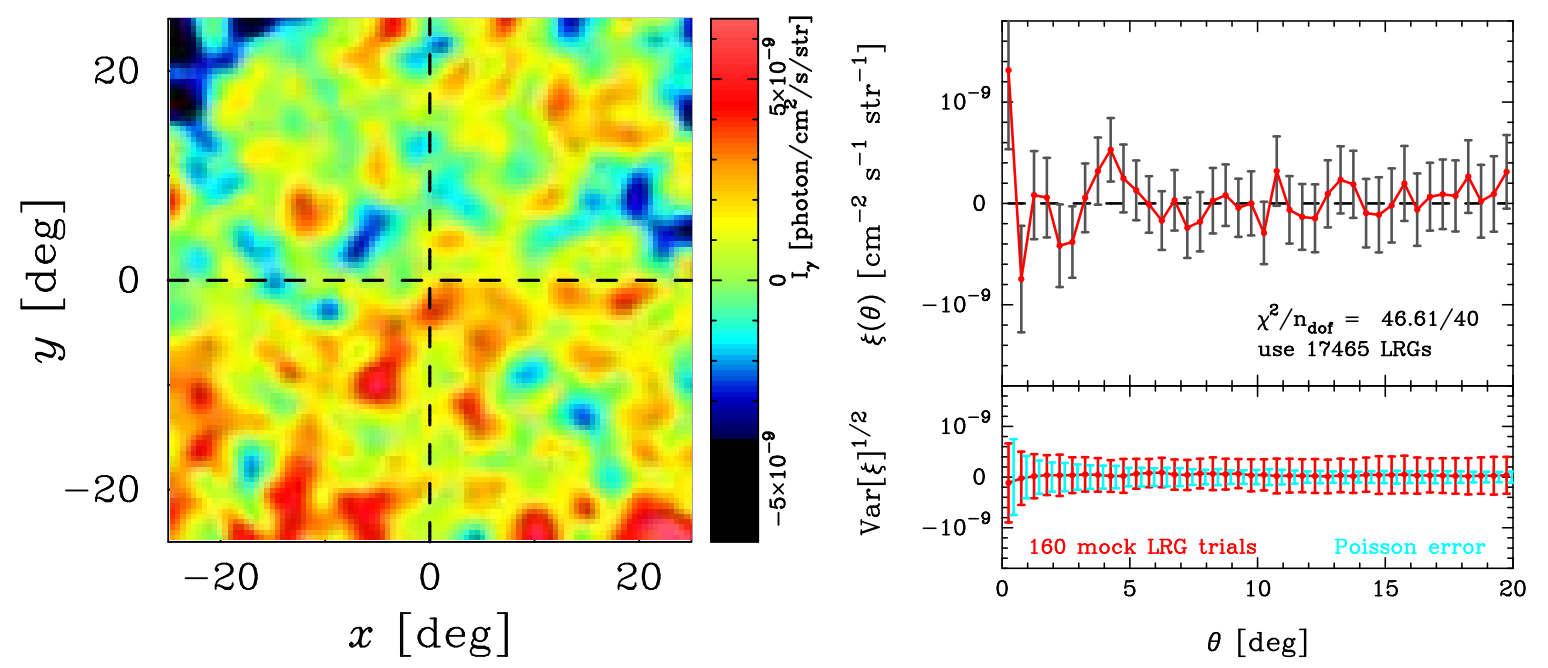

FIG. 2. The cross-correlation of the EGB with LRGs. Left: the stacked image of EGB photons around 17,465 LRGs. The color coordinate represents the EGB intensity in the energy range of $1-500 \mathrm{GeV}$. Right: the cross-correlation function as a function of separation angle. In the top panel, the red line represents our measurement and the gray error bars correspond to the statistical uncertainty estimated from 160 mock catalogs. The bottom panel shows the contribution to the statistical uncertainty from sample variance (red) and Poisson photon noise (cyan) separately.

each randomized photon map. We then repeat the crosscorrelation analysis for two different sets of catalogues, i.e., 160 randomized gamma-ray maps and the observed galaxy catalogue or the observed gamma-ray map and the 160 mock galaxy catalogues. We estimate the statistical error associated with the spatial clusterings of LRGs and the photon noise by summing these two contributions. Note that our estimate of covariance includes the sampling variance of LRGs because we use independent mock LRGs of which spatial clustering pattern is closely matched to that of the real LRGs. We calculate Eq. (16) on the assumption that the LRGs and the EGB do not correlate each other. For our analysis, it is sufficient to verify whether the LRGs correlate with the EGB or not.

The cross-correlation estimator [Eq. (15)] is dependent on the model for the astrophysical foreground emission of our own Galaxy. We therefore use variants of the foreground emission models provided by the Fermi collaboration to assess the impacts on the estimated EGB. We work with 35 different Galactic diffuse models from Ref. [50]. The details of these analyses are summarized in Section VIA.

\section{RESULT}

Figure 2 summarizes the result of our cross-correlation analyses. In the left panel, we show a stacked image of the EGB intensity around the angular positions of LRGs. In the right panel, we present the cross-correlation function as a function of separation angle $\theta$. In the upper portion of the right panel, the red line shows the measured signal and the gray error bars show the statistical error estimated from a set of mock catalogs. In the lower por- tion of the right panel, we show the contributions to the total statistical uncertainty from photon noise and sample variance separately, and find that the photon noise is comparable to the sample variance of LRGs in our measurement.

In order to quantify the significance of the measured cross-correlation signal with respect to the statistical error, we use the $\chi^{2}$ statistics defined by,

$$
\chi^{2}=\sum_{i, j} \xi\left(\theta_{i}\right) C_{i j}^{-1} \xi\left(\theta_{j}\right)
$$

where $\boldsymbol{C}^{-1}$ denotes the inverse covariance matrix estimated from the randomized realization shown in Section [IV] In our analysis, the number of degrees of freedom is 40 . The resulting value of $\chi^{2} / n_{\text {dof }}$ is $46.61 / 40$, which implies a good fit without DM annihilation.

We are now able to use the null detection of the crosscorrelation to place constraints on the DM annihilation cross-section. For this purpose, we use the maximum likelihood analysis. We assume that the data vector $\boldsymbol{D}$ is well approximated by the multivariate Gaussian distribution with covariance $\boldsymbol{C}$. In this case, $\chi^{2}$ statistics (log-likelihood) is given by,

$$
\chi^{2}(\boldsymbol{p})=\sum_{i, j}\left(D_{i}-\mu_{i}(\boldsymbol{p})\right) C_{i j}^{-1}\left(D_{j}-\mu_{j}(\boldsymbol{p})\right),
$$

where $\boldsymbol{\mu}(\boldsymbol{p})$ is the theoretical template for the set of parameters of interest. In this paper, we use the halo model approach described in Section $\amalg A$ to calculate the theoretical prediction. As parameters of interest $\boldsymbol{p}$, we simply consider the DM particle mass and the annihilation cross-section, $m_{\mathrm{dm}}$ and $\langle\sigma v\rangle$. The data vector $\boldsymbol{D}$ consists 


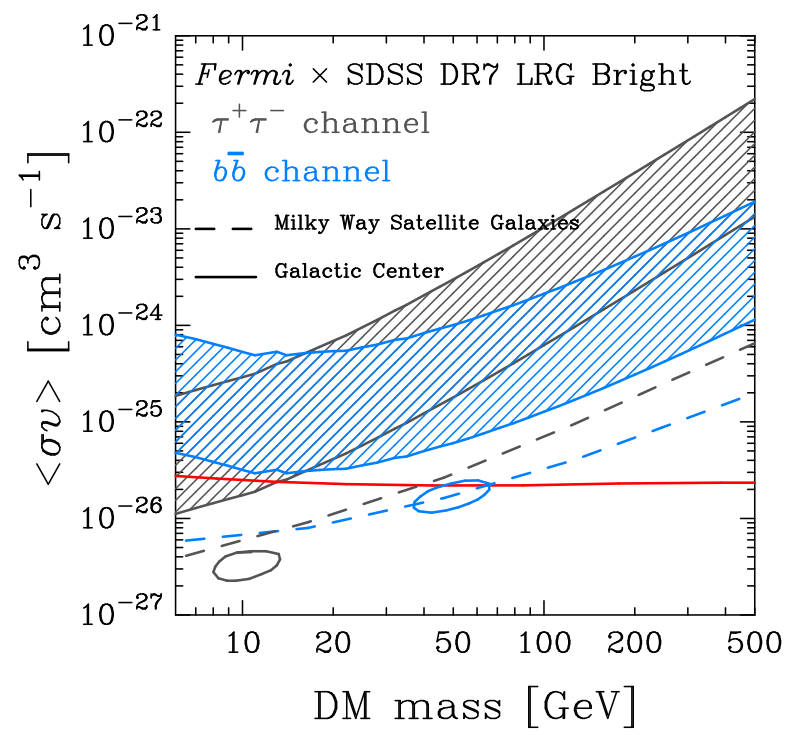

FIG. 3. Shown are 95\%C.L. upper limits on the DM annihilation cross-section. The two shaded bands are the limits derived from our cross-correlation analysis of the EGB with LRGs, showing two annihilation channels, $b \bar{b}$ (cyan) and $\tau^{+} \tau^{-}$(gray). For each channel, the shaded band represents a conservative estimate of the theoretical uncertainty due to DM substructure. For comparison, we also plot constraints from Ref. [2] derived from staked satellites galaxies of the Milky Way (dashed), and regions of best fit from studies of the Milky Way Galactic center from Ref. 6] (closed circles), respectively. The red line shows the canonical cross-section expected for thermal relic DM [51].

of the ten measured cross-correlation amplitudes in the range of $\theta=[0,5]$ degree as,

$$
D_{i}=\left\{\xi\left(\theta_{1}\right), \xi\left(\theta_{2}\right), \ldots, \xi\left(\theta_{10}\right)\right\}
$$

where $\theta_{i}$ is the $i$-th angular separation bin. The inverse covariance matrix $\boldsymbol{C}^{-1}$ includes both the statistical error owing to the spatial clusterings of LRGs and the photon Poisson error. We consider the $95 \%$ confidence level of posterior distribution function of parameters. This is given by the contour line in the two dimensional space $\left(m_{\mathrm{dm}}\right.$ and $\left.\langle\sigma v\rangle\right)$, which is defined as

$$
\Delta \chi^{2}(\boldsymbol{p})=\chi^{2}(\boldsymbol{p})-\chi^{2}(\boldsymbol{\mu}=0)=6.17 .
$$

As discussed in Section IIA the choice of boost factor $b_{s h}$ affects the theoretical predictions significantly, by a factor of about ten. We therefore derive constraints based on an optimistic scenario adopting Ref. 24], and a conservative scenario adopting Ref. [25].

Figure 3 shows the result of our likelihood analysis on the DM parameter space $m_{\mathrm{dm}}$ and $\langle\sigma v\rangle$. We plot the constraints for two representative particle physics model, the $\tau^{+} \tau^{-}$channel and the $b \bar{b}$ channel. The shaded band is derived from the two extreme assumptions of the boost factor, and thus the true bound is expected to lie in the shaded region. The constraint for the large boost factor model [24] is significantly stronger, as expected. For reference, the red line indicates the canonical cross-section of for a thermally produced DM [51].

\section{SYSTEMATIC UNCERTAINTIES}

In this section, we investigate possible systematic uncertainties in our cross-correlation analysis. We consider two contributions: (i) uncertainty in modeling the Galactic gamma-rays foregrounds, and (ii) possible correlation due to gamma-ray emission from star formation in LRGs.

\section{A. Galactic model template}

The main contribution to the $E_{\gamma}>100 \mathrm{MeV}$ gammaray sky arises from diffuse emissions produced by interactions of Galactic cosmic rays with the gas and radiation fields in the Galaxy. Estimates of the EGB are crucially affected by this Galactic diffuse emission which need to be subtracted from the observed gamma-ray intensity.

Determining the Galactic diffuse emission requires treatments of multiple processes. Cosmic-ray interactions with the interstellar gas produce neutral pions which subsequently decay to gamma-ray pairs. Interactions with gas also give rise to bremsstrahlung emission, which can be a significant contribution in gas-rich environments of the Galaxy. Finally, cosmic-ray electrons up-scatter low-energy photons to gamma-ray energies via the inverse-Compton process. At the same time, cosmic rays propagate diffusively or rectilinearly in the magnetic field of the Milky Way. The final prediction therefore depends on the source distribution of cosmic rays, the injection spectra and composition of cosmic rays, the distribution of interstellar gas, light, and magnetic field in the Milky Way, and the assumed propagation model.

In order to estimate the possible uncertainties in such complex phenomena, we follow Ref. [52 and generate multiple Galactic gamma-ray foreground models using the GALPROP (version 54) propagation code 53 55]. In Ref. [52], a total of 128 models were explored by varying the following parameters: the cosmic-ray source distribution, the cosmic-ray confinement region, and assumptions affecting the interstellar gas distribution. For the cosmicray source distribution, four distributions were explored: the supernova remnant distribution of Ref. 56], the Ostar distribution of Ref. [57], and the pulsar distributions of Refs. [58] and [59]. For the cosmic-ray confinement region, cylindrical volumes with heights $z=4,6,8$, and 10 $\mathrm{kpc}$, and radii $R=20$ and $30 \mathrm{kpc}$ were explored. For the interstellar gas distribution, two assumptions for the optical depth correction to the atomic hydrogen component $\left(T_{s}=150 \mathrm{~K}\right.$ and $\left.10^{5} \mathrm{~K}\right)$, and two values for the cut at which dust emission is no longer used to correct for missing neutral gas $(E(B-V)=2$ and 5 mags) were explored. For each of these models, parameters affecting the cosmic ray and gas are varied and fitted to Fermi-LAT data. 

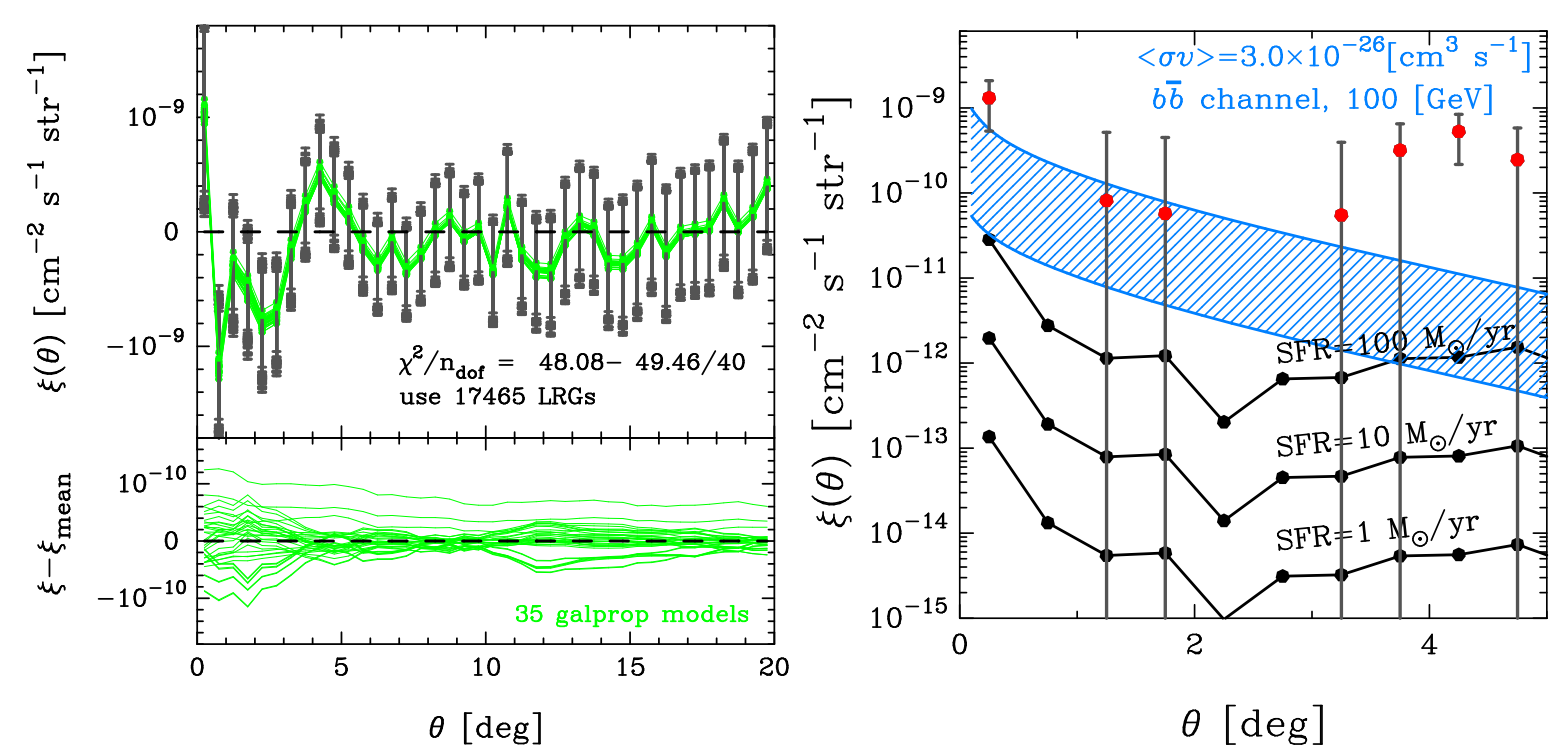

FIG. 4. Systematic uncertainties in our cross-correlation analysis. Left: the results of cross-correlation analyses for EGB data obtained using 35 different Galactic gamma-ray foreground templates. In the top portion, the green lines and grey error bars show the measured cross-correlation signals $\xi$ for a given galactic template. The green lines are virtually indistinguishable. The bottom portion shows the differences in $\xi$ from $\xi_{\text {mean }}$, where $\xi_{\text {mean }}$ represents the mean cross-correlation signal over the data set derived from 35 different Galactic gamma-ray foreground templates. Right: the expected correlation from star-forming gamma-rays in LRGs. The three solid lines show the cases with star formation rates of 1, 10, and $100 M_{\odot} /$ yr. For comparison, the shaded region represents the expected cross-correlation signals calculated from our halo model in the case of annihilating DM (into $b \bar{b}$ ) with the mass of $100 \mathrm{GeV}$ and with a thermal canonical cross-section. We also plot the measured signal (but only positive) as shown in Fig. 2 by the red point with gray error bar.

The fitted parameters include those setting the injected cosmic-ray nuclei and electron spectral shapes and normalizations, the diffusion coefficient, as well as conversion factors between $\mathrm{CO}$ and molecular hydrogen. We refer the reader to Ref. [52] for details of the parameters and fits.

We simulate a total of 35 among these 128 models. Specifically, we simulate all 32 models that use the pulsar distribution of Ref. [58] as a proxy for the cosmic-ray spatial distribution. In addition, we simulate one additional model for each of the other three cosmic-ray source distributions, all for the same inputs parameters $z=6$ $\mathrm{kpc}, R=30 \mathrm{kpc}, T_{s}=150 \mathrm{~K}$, and $E(B-V)=2 \mathrm{mag}$ cut. We use these 35 model templates and repeat the cross-correlation analysis described in Section IV] We estimate not only the cross-correlation signal but also the statistical error for each galactic model template. We therefore obtain 35 different binned signals $(\xi)$ as functions of angular separation.

The left panel of Fig. 4 shows the results of the crosscorrelation analyses using the model templates. In the top panel, we plot all the cross-correlation signals with their respective statistical errors. The green lines appear virtually indistinguishable. Clearly, the differences among model templates are significantly smaller than the statistical error. The resulting $\chi^{2}$ ranges from 48.08 to 49.46 with 40 data bins. The bottom panel shows the variance of $\xi$ among the 35 trials. The typical ampli- tude of variance due to the uncertainty in the Galactic model templates is $\sim 1 \times 10^{-10} \mathrm{~cm}^{-2} \mathrm{~s}^{-1} \mathrm{str}^{-1}$, which is $\sim 10$ times smaller than the current statistical uncertainty (see the right panel in Figure 2). Therefore, we conclude that the modeling of the Galactic gamma-ray distribution does not significantly affect our result. This is a result of the SDSS survey region being at relatively high latitudes where the Galactic diffusion emission models are relatively better modeled than, e.g., along the Galactic plane [52].

\section{B. Contribution from astrophysical sources}

There is another important uncertainty in studying DM annihilation with cross-correlation: gamma-rays emitted from star-forming or/and radio galaxies contaminating the cross-correlation measurement. Generally, astrophysical sources are expected to contribute considerably to the EGB [12, 60 62]. In order to estimate the possible correlation of LRGs and gamma rays from astrophysical sources, we utilize the observed LRG distribution and known scaling relations between the gamma-ray intensity and star-forming activity.

We consider several fixed values of the star-forming rate (SFR) of the LRGs, and assign gamma-ray luminosity to each LRG according to the gamma ray to SFR scaling relation given in Ref [61]. The model yields 
correlations of the LRGs and astrophysical gamma rays that are equal to the auto correlation of the LRGs multiplied by the gamma-ray intensity of each astrophysical source. Hence, the contribution from astrophysical sources $\xi_{\text {ast }}(\theta)$ is,

$$
\xi_{\text {ast }}(\operatorname{SFR}, \theta) \simeq I_{\gamma, \text { ast }}(\operatorname{SFR}) \xi_{\mathrm{LRG}}(\theta),
$$

where $I_{\gamma \text {,ast }}(\mathrm{SFR})$ represents the gamma-ray intensity as a function of SFR expected from the scaling relation and $\xi_{\text {LRG }}(\theta)$ is angular correlation function of LRGs. The right panel in Fig. 4 4 shows three different cases of $\xi_{\text {ast }}(\theta)$ with SFR of 1,10 , and $100 M_{\odot} /$ yr. The SFR distribution of LRG systems has been studied in Ref [63] and the typical value ranges from 0.1 to $1 M_{\odot} /$ yr. Since we do not take into account the off-centering effect of astrophysical sources in the LRG host halo or the smearing effect of gamma-ray PSF, our estimation of $\xi_{\text {ast }}$ with Eq. (22) is conservative. Clearly, the contribution from star-forming galaxies is small, and thus does not affect our cross-correlation analysis of the EGB with LRGs.

Similar results are obtained in the case of radio galaxies. Ref. 64] presents a possible correlation of radio galaxies and LRGs, and also shows that the typical radio luminosity of LRGs at $1.4 \mathrm{GHz}$ is $L_{1.4 \mathrm{GHz}} \sim 4 \times$ $10^{22} \mathrm{~W} \mathrm{~Hz}^{-1}$. Using the scaling relation in Ref. 61, this corresponds to an expected gamma-ray intensity of $\sim 3 \times 10^{40} \mathrm{erg} \mathrm{s}^{-1}$. Scaling to SFR, this is equivalent to $\sim 20 M_{\odot} /$ yr. Hence, the contribution from radio galaxies is also not significant.

\section{CONCLUSION AND DISCUSSION}

In this paper, we have performed a cross-correlation analysis of the EGB with LRGs. Using gamma-ray data from the Fermi satellite and 17,465 LRGs in the SDSS catalog, we find that the cross-correlation signal is consistent with null detection (Fig. 2). By using up-to-date theoretical models based on recent galaxy clustering and weak lensing studies, we estimate the statistical errors from a combination of real data and a large set of mock observations. We derive constraints on the DM annihilation cross-section, considering different DM annihilation channels and different models of the amount of substructures in DM halos. The DM annihilation cross-section must be smaller than $\langle\sigma v\rangle<10^{-25}-10^{-23} \mathrm{~cm}^{3} \mathrm{~s}^{-1}$ for a $100 \mathrm{GeV}$ DM, depending on the assumed parameters and annihilation channel (Fig. 3). The constraint improves for smaller DM mass.

We have further investigated two systematic uncertainties in our DM constraint (Figure 4). The first is due to the uncertainty in the Galactic gamma-ray foreground emission model. In order to evaluate the overall error, we utilize 35 different Galactic diffuse emission templates produced by the GALPROP code and repeat the cross-correlation analysis for the EGB data set derived by each template. The variation in the correlation signal due to differences in the Galactic templates is about ten times smaller than the statistical error of current observations. Although the template uncertainties depend on sky position, the systematic uncertainty is thus expected to be unimportant even in future galaxy surveys with sky coverages of 20,000 square degrees that would reduce the statistical error by a factor of $\sim \sqrt{20000 / 7200}=1.7$. The second source of systematic error is the possible correlation due to gamma rays emitted from astrophysical sources associated with LRGs. We estimate this contamination by using empirical scaling relations observed between the gamma-ray luminosity and star-forming activities of nearby galaxies [61]. The estimated correlation is $20-30$ times smaller than the expected signal of DM annihilation with DM mass of $100 \mathrm{GeV}$. Therefore, we conclude that LRGs are an ideal target for the statistical detection of DM annihilation by means of crosscorrelation analysis.

Encouraged by the results of our systematic uncertainty investigations, we forecast the improvement expected with upcoming galaxy surveys, such as the LSST with a sky coverage of 20,000 square degrees. When we simply assume that the statistical uncertainty is reduced by a factor of $\sqrt{20000 / 7200}$, the expected constraints on $\langle\sigma v\rangle$ would reach $7.8-130 \times 10^{-26} \mathrm{~cm}^{3} \mathrm{~s}^{-1}$ for the $b \bar{b}$ channel and $3.7-62 \times 10^{-25} \mathrm{~cm}^{3} \mathrm{~s}^{-1}$ for the $\tau^{+} \tau^{-}$ channel, both for a $100 \mathrm{GeV}$ DM. For a lighter DM motivated by the Galactic center excess (e.g., [5, 6, 65 61]), the constraints would reach $2.84-42.1 \times 10^{-26} \mathrm{~cm}^{3} \mathrm{~s}^{-1}$ for the $b \bar{b}$ channel (assuming $40 \mathrm{GeV}$ mass) and $1.02-$ $17.17 \times 10^{-26} \mathrm{~cm}^{3} \mathrm{~s}^{-1}$ for the $\tau^{+} \tau^{-}$(assuming $10 \mathrm{GeV}$ mass). Therefore, future large-area galaxy surveys will test the DM origin hypothesis of the Galactic center excess. Note that the expected constraints by LRGs in 20,000 square degrees would be competitive to the recent constraints derived from the cross-correlation analysis with local galaxies [72], and it would be $\sim 10$ times tighter than the current limits obtained from the energy spectrum of EGB (the conservative limit shown in Ref [73]).

We have shown that the LRGs are a promising target to search for DM annihilation. The single largest uncertainty is the amount of substructures in DM halos, which significantly affects our theoretical model of gamma-ray emission. Previous works have cultivated theoretical understanding of the effect of halo substructures on the DM annihilation signal [26, 74 76 , but there has been limited information about the amount of substructures in cluster-sized DM halos. Further observations will help understand the properties and abundance of halo substructure to calibrate theoretical models. Gravitational lensing analysis in nearby clusters [77], and stacking analysis of member galaxies in clusters [78, 79], would be the first base of such studies. 


\section{ACKNOWLEDGMENTS}

The authors thank Shin'ichiro Ando, Stefano Camera and Matteo Viel for useful discussions and comments on the manuscript. M.S. is supported by Research Fellow- ships of the Japan Society for the Promotion of Science (JSPS) for Young Scientists. N.Y. acknowledges financial support from JST CREST. Numerical computations presented in this paper were in part carried out on the general-purpose $\mathrm{PC}$ farm at Center for Computational Astrophysics, CfCA, of National Astronomical Observatory of Japan.
[1] G. Jungman, M. Kamionkowski, and K. Griest, Phys.Rept. 267, 195 (1996), hep-ph/9506380

[2] M. Ackermann et al. (Fermi-LAT Collaboration), $\quad$ Phys.Rev. D89, 042001 (2014) arXiv:1310.0828 [astro-ph.HE]

[3] M. R. Buckley, E. Charles, J. M. Gaskins, A. M. Brooks, A. Drlica-Wagner, P. Martin, and G. Zhao, Phys.Rev., 102001 (2015), arXiv:1502.01020 [astro-ph.HE]

[4] M. Ackermann et al. (Fermi-LAT),

(2015), arXiv:1503.02641 [astro-ph.HE]

[5] K. N. Abazajian and M. Kaplinghat, $\quad$ Phys.Rev. D86, 083511 (2012) arXiv:1207.6047 [astro-ph.HE]

[6] K. N. Abazajian, N. Canac, S. Horiuchi, and M. Kaplinghat, Phys. Rev. D90, 023526 (2014), arXiv:1402.4090 [astro-ph.HE]

[7] S. Camera, M. Fornasa, N. Fornengo, and M. Regis, Astrophys.J. 771, L5 (2013) arXiv:1212.5018 [astro-ph.CO],

[8] N. Fornengo and M. Regis, Frontiers in Physics 2, 6 (2014) arXiv:1312.4835 [astro-ph.CO],

[9] S. Ando, A. Benoit-Lévy, and E. Komatsu, Phys.Rev. , 023514 (2014), arXiv:1312.4403.

[10] S. Ando, JCAP 1410, 061 (2014) arXiv:1407.8502 [astro-ph.CO],

[11] S. Camera, M. Fornasa, N. Fornengo, and M. Regis, JCAP 6, 029 (2015), arXiv:1411.4651.

[12] M. Ajello, D. Gasparrini, M. Sánchez-Conde, G. Zaharijas, M. Gustafsson, J. Cohen-Tanugi, C. D. Dermer, Y. Inoue, D. Hartmann, M. Ackermann, K. Bechtol, A. Franckowiak, A. Reimer, R. W. Romani, and A. W. Strong, Astrophys.J.Lett. 800, L27 (2015), arXiv:1501.05301 [astro-ph.HE]

[13] M. Fornasa and M. A. SánchezConde, $\quad$ Phys. Rept. 598, 1 (2015) arXiv:1502.02866 [astro-ph.CO].

[14] J.-Q. Xia, A. Cuoco, E. Branchini, and M. Viel, Astrophys.J.Suppl. 217, 15 (2015), arXiv:1503.05918

[15] N. Fornengo, L. Perotto, M. Regis, and S. Camera, Astrophys.J.Lett. 802, L1 (2015), arXiv:1410.4997.

[16] A. Cuoco, J.-Q. Xia, M. Regis, E. Branchini, N. Fornengo, and M. Viel, (2015), arXiv:1506.01030 [astro-ph.HE]

[17] R. Gilmore, R. Somerville, J. Primack, and A. Dominguez, Mon.Not.Roy.Astron.Soc. 422, 3189 (2012), arXiv:1104.0671 [astro-ph.CO].

[18] M. Cirelli, G. Corcella, A. Hektor, G. Hutsi, M. Kadastik, et al., JCAP 1103, 051 (2011) arXiv:1012.4515 [hep-ph]

[19] J. L. Tinker, A. V. Kravtsov, A. Klypin, K. Abaza- jian, M. S. Warren, et al., Astrophys.J. 688, 709 (2008) arXiv:0803.2706 [astro-ph]

[20] J. L. Tinker, B. E. Robertson, A. V. Kravtsov, A. Klypin, M. S. Warren, et al., Astrophys.J. 724, 878 (2010), arXiv:1001.3162 [astro-ph.CO]

[21] S. Ando and E. Komatsu, Phys.Rev. D87, 123539 (2013) arXiv:1301.5901 [astro-ph.CO]

[22] J. F. Navarro, C. S. Frenk, and S. D. White, Astrophys.J. 490, 493 (1997) arXiv:astro-ph/9611107 [astro-ph]

[23] F. Prada, A. A. Klypin, A. J. Cuesta, J. E. Betancort-Rijo, and J. Primack, Mon. Not. Roy. Astron. Soc. 428, 3018 (2012) arXiv:1104.5130 [astro-ph.CO]

[24] L. Gao, C. Frenk, A. Jenkins, V. Springel, and S. White, Mon.Not.Roy.Astron.Soc. 419, 1721 (2012), arXiv:1107.1916 [astro-ph.CO]

[25] M. A. Sánchez-Conde and F. Prada, Mon. Not. Roy. Astron. Soc. 442, 2271 (2014) arXiv:1312.1729 [astro-ph.CO]

[26] R. Bartels and S. Ando, arXiv:1507.08656 [astro-ph.CO]

$[27]$ B. A. Reid and D. N. Spergel, Astrophys.J. 698, 143 (2009) arXiv:0809.4505 [astro-ph].

[28] D. A. Wake, R. K. Sheth, R. C. Nichol, C. M. Baugh, J. Bland-Hawthorn, et al., Mon.Not.Roy.Astron.Soc. 387, 1045 (2008) arXiv:0802.4288 [astro-ph]

[29] C. Hikage, R. Mandelbaum, M. Takada, and D. N. Spergel, Mon.Not.Roy.Astron.Soc. 435, 2345 (2013) arXiv:1211.1009 [astro-ph.CO]

[30] D. N. Limber, Astrophys.J. 119, 655 (1954).

[31] M. Fukugita, T. Ichikawa, J. Gunn, M. Doi, K. Shimasaku, et al., Astron.J. 111, 1748 (1996)

[32] J. Gunn et al. (SDSS), Astron.J. 116, 3040 (1998) arXiv:astro-ph/9809085 [astro-ph]

[33] J. E. Gunn et al. (SDSS), Astron.J. 131, 2332 (2006) arXiv:astro-ph/0602326 [astro-ph]

[34] R. Lupton et al. (SDSS), ASP Conf.Ser. 238, 269 (2001), arXiv:astro-ph/0101420 [astro-ph]

[35] C. Stoughton et al. (SDSS), Astron.J. 123, 485 (2002)

[36] J. R. Pier, J. A. Munn, R. B. Hindsley, G. Hennessy, S. M. Kent, et al., Astron.J. 125, 1559 (2003), arXiv:astro-ph/0211375 [astro-ph]

[37] Z. Ivezic et al. (SDSS), Astron.Nachr. 325, 583 (2004) arXiv:astro-ph/0410195 [astro-ph]

[38] D. W. Hogg, D. P. Finkbeiner, D. J. Schlegel, and J. E. Gunn, Astron.J. 122, 2129 (2001) arXiv:astro-ph/0106511 [astro-ph]

[39] J. A. Smith et al. (SDSS), Astron.J. 123, 2121 (2002) 
arXiv:astro-ph/0201143 [astro-ph].

[40] D. Tucker et al. (SDSS), Astron.Nachr. 327, 821 (2006) arXiv:astro-ph/0608575 [astro-ph].

[41] D. J. Eisenstein et al. (SDSS), Astron.J. 122, 2267 (2001) arXiv:astro-ph/0108153 [astro-ph].

[42] M. A. Strauss et al. (SDSS), Astron.J. 124, 1810 (2002) arXiv:astro-ph/0206225 [astro-ph].

[43] G. T. Richards et al. (SDSS), Astron.J. 123, 2945 (2002), arXiv:astro-ph/0202251 [astro-ph].

[44] M. R. Blanton, R. H. Lupton, F. Miller Malley, N. Young, I. Zehavi, et al., Astron.J. 125, 2276 (2003) arXiv:astro-ph/0105535 [astro-ph].

[45] K. N. Abazajian et al. (SDSS), Astrophys.J.Suppl. 182, 543 (2009), arXiv:0812.0649 [astro-ph]

[46] E. A. Kazin et al. (SDSS), Astrophys.J. 710, 1444 (2010), arXiv:0908.2598 [astro-ph.CO]

[47] J.-Q. Xia, A. Cuoco, E. Branchini, M. Fornasa, and M. Viel, Mon.Not.Roy.Astron.Soc. 416, 2247 (2011), arXiv:1103.4861 [astro-ph.CO].

[48] M. Su, T. R. Slatyer, and D. P. Finkbeiner, $\quad$ Astrophys.J. 724, 1044 (2010), arXiv:1005.5480 [astro-ph.HE]

[49] M. Shirasaki, S. Horiuchi, and N. Yoshida, Phys.Rev. D90, 063502 (2014), arXiv:1404.5503 [astro-ph.CO].

[50] M. Ackermann et al. (FermiLAT), Astrophys. J. 750, 3 (2012), arXiv:1202.4039 [astro-ph.HE].

[51] G. Steigman, B. Dasgupta, and J. F. Beacom, $\quad$ Phys. Rev. D 86, 023506 (2012), arXiv:1204.3622 [hep-ph]

[52] M. Ackermann et al. (FermiLAT), Astrophys.J. 750, 3 (2012) arXiv:1202.4039 [astro-ph.HE]

[53] A. Strong, I. V. Moskalenko, and O. Reimer, International Cosmic Ray Conference 4, 52 (1999), astro-ph/9906229.

[54] A. W. Strong and I. V. Moskalenko, International Cosmic Ray Conference 4, 255 (1999), astro-ph/9906228.

[55] I. V. Moskalenko and A. W. Strong, Astrophysics and Space Science 272, 247 (2000), astro-ph/9908032

[56] G. L. Case and D. Bhattacharya, Astrophys. J. 504, 761 (1998) arXiv:astro-ph/9807162 [astro-ph].

[57] L. Bronfman, S. Casassus, J. May, and L. A. Nyman, Astron. Astrophys. 358, 521 (2000), arXiv:astro-ph/0006104 [astro-ph].

[58] D. $\quad$ R. L Lorimer et al., Mon. Not. Roy. Astron. Soc. 372, 777 (2006), arXiv:astro-ph/0607640 [astro-ph].

[59] I. Yusifor and I. Kucuk,
Astron. Astrophys. 422, 545 (2004) arXiv:astro-ph/0405559 [astro-ph]

[60] Y. Inoue, $\quad$ Astrophys.J. 733, 66 (2011) arXiv:1103.3946 [astro-ph.HE]

[61] M. Ackermann et al. (Fermi LAT Collaboration), arXiv:1206.1346 [astro-ph.HE]

[62] M. Di Mauro, F. Calore, F. Donato, M. Ajello, and L. Latronico, Astrophys.J. 780, 161 (2014) arXiv:1304.0908 [astro-ph.HE]

[63] X.-F. Deng, Y.-Q. Chen, and P. Jiang, Mon.Not.Roy.Astron.Soc. 417, 453 (2011)

[64] J. A. Hodge, G. R. Zeimann, R. H. Becker, and R. L. White, Astron.J. 138, 900 (2009), arXiv:0907.1081.

[65] L. Goodenough and D. Hooper, (2009), arXiv:0910.2998 [hep-ph]

[66] D. Hooper and L. Goodenough, Phys.Lett. B697, 412 (2011), arXiv:1010.2752 [hep-ph].

[67] D. Hooper and T. Phys.Rev. D84, 123005 (2011) arXiv:1110.0006 [astro-ph.HE]

[68] A. Boyarsky, D. Malyshev, and O. Ruchayskiy, Phys.Lett. B705, 165 (2011), arXiv:1012.5839 [hep-ph]

[69] C. Gordon and O. Phys.Rev. D88, 083521 (2013) arXiv:1306.5725 [astro-ph.HE]

[70] T. Daylan, D. P. Finkbeiner, D. Hooper, T. Linden, S. K. N. Portillo, et al., (2014), arXiv:1402.6703 [astro-ph.HE]

[71] K. N. Abazajian, N. Canac, S. Horiuchi, M. Kaplinghat, and A. Kwa, JCAP 1507, 013 (2015) arXiv:1410.6168 [astro-ph.HE]

[72] M. Regis, J.-Q. Xia, A. Cuoco, E. Branchini, N. Fornengo, and M. Viel, Physical Review Letters 114, 241301 (2015), arXiv:1503.05922

[73] M. Ackermann et al. (FermiLAT), JCAP 1509, 008 (2015) arXiv:1501.05464 [astro-ph.CO]

[74] M. Kuhlen, J. Diemand, and P. Madau, Astrophys.J. 686, 262 (2008), arXiv:0805.4416.

[75] M. Kamionkowski, S. M. Koushiappas, and M. Kuhlen, Phys.Rev. , 043532 (2010) arXiv:1001.3144 [astro-ph.GA]

[76] J. Zavala and N. Afshordi, Mon.Not.Roy.Astron.Soc. 441, 1329 (2014) arXiv:1311.3296

[77] N. Okabe, Y. Okura, and T. Futamase, Astron.J. 713, 291 (2010), arXiv:1001.2402

[78] R. Li, H. Shan, H. Mo, J.-P. Kneib, X. Yang, W. Luo, F. C. van den Bosch, T. Erben, B. Moraes, and M. Makler, Mon.Not.Roy.Astron.Soc. 438, 2864 (2014), arXiv:1311.6523 [astro-ph.CO]

[79] M. Shirasaki, $\quad$ Astrophys.J. 799, 188 (2015) arXiv:1407.5350 